\title{
Defined Imaging
}

National Cancer Institute

\section{Source}

National Cancer Institute. Defined Imaging. NCI Thesaurus. Code C93370.

An activity defined at the global library level whose intention is to obtain pictures of the interior or exterior of the body usually for diagnostic reasons. 